\title{
Retrofit da Máquina de Circulação Extracorpórea com dispositivo IoMT
}

\section{Retrofit of Extracorporeal Circulation Machine with IoMT device}

\author{
Correia FFB, Gonçalves DR, Pacheco CAD, Ramos MH e Leão TF* \\ *Instituto Federal de Educação, Ciência e Tecnologia de São Paulo, São Paulo, Brasil.
}

\begin{abstract}
Resumo. A evolução de tecnologias disruptivas na área médica toma proporções significativas na transformação do setor. O termo IoMT (Internet of Medical Things) define como essa transformação se expande e traz benefícios de performance, maximizando a interação entre máquinas e pessoas. Nesse contexto, o presente trabalho tem por objetivo aprimorar uma máquina de circulação extracorpórea (CEC) mediante a aplicação de tecnologias de IoMT, melhorando os procedimentos de monitoramento e controle de operação do conjunto com a implementação de um estimador de pressão e vazão da bomba. No desenvolvimento do projeto foi possível implementar um aplicativo móvel que se comunica com a placa microcontrolada para controlar o motor da máquina, bem como um estimador com interpolação para calcular e aproximar os valores da bomba. Utilizou-se ferramentas de desenvolvimento por etapas com testes de funcionamento modulares e, assim, identificou-se melhorias consideráveis de cálculo dos valores por meio de comparações entre a simulação do ambiente realizada no Proteus e o programa anterior da máquina, tal como a comparação com o estudo elaborado na tese que inspirou este trabalho. Por fim, verificou-se o teste de funcionamento, integrando todas as partes funcionais da máquina em ambiente simulado e, dessa forma, validando a melhoria de operação remota da máquina proposta neste projeto. $\mathrm{O}$ resultado permite futuros trabalhos baseado no arcabouço estudado, na implementação e expansão deste projeto com a construção de controles modernos, melhorias do aplicativo móvel e elaboração de placa eletrônica própria para a máquina.
\end{abstract}

Palavras-chave. IoMT; Máquina de Circulação Extracorpórea; Estimador; Aplicativo Móvel.

\begin{abstract}
The evolution of disruptive technologies in the medical field takes significant proportions in the transformation of the sector. The term IoMT (Internet Of Medical Things) defines how this transformation expands and brings performance benefits, maximizing the interaction between machines and people. In this context, the present work improves an extracorporeal circulation machine through the application of IoMT technologies, improving the procedures for monitoring and controlling the operation of the set with the implementation of an estimator of pump pressure and flow. In the development of the project, it was possible to implement a mobile application that communicates with the microcontrolled plate to control the
\end{abstract}


machine's motor, as well as an interpolation estimator to calculate and approximate the pump values. The development were made in stages with modular functional tests and considerable improvements in the calculation of values were identified through comparisons between the environment simulation performed on Proteus and the previous machine program, such as the comparison with the study elaborated on the thesis that inspired this work. Finally, the function test was verified, integrating all the functional parts of the machine in a simulated environment and, thus, validating the remote operation improvement of the machine proposed in this project. The legacy left here allows this study to serve as a framework for future work, in the implementation and expansion of this project with the construction of modern controls, improvements of the mobile application and the elaboration of an electronic plate suitable for the machine.

Keywords. IoMT; Extracorporeal Circulation Machine; Estimator; Mobile Application.

Introdução. Mesmo com o advento da automação e sua crescente evolução, grande parte dos hospitais não usufruem ativamente dos benefícios que essa transformação trouxe para a atualidade. Melhorias consideráveis podem ser implementadas para a prevenção e gerenciamento de doenças e processos hospitalares.

O conceito de IoMT é uma parte crítica da transformação digital da assistência médica, pois permite o surgimento de novos modelos de negócios e permite mudanças nos processos de trabalho, melhorias de produtividade, contenção de custos e experiências aprimoradas dos clientes (Dimitrov, 2016). Por meio dessa tecnologia é possível incluir dispositivos portáteis com acesso à internet de alta velocidade que monitoram constantemente indicadores de saúde dos pacientes ou que auxiliam a área médica em cirurgias de alto risco ou em consultas clínicas genéricas. Segundo o Grand View Research (2019), o IoT aplicado ao mercado Healthcare tem previsão de expansão, chegando a atingir 534,3 bilhões de dólares até 2025, expandindo-se em progressão geométrica $19,9 \%$ durante o período.

Com a finalidade de aplicar esses conceitos em um trabalho voltado para o cenário brasileiro, $\mathrm{O} \mathrm{LAV}^{1}$ disponibilizou uma máquina de circulação extracorpórea para que os dados captados pelos sensores do mesmo sejam monitorados e controlados em apenas um ponto focal da sala por um dispositivo remoto, considerando a criticidade de se trabalhar com um sistema de suporte a vida em ambiente cirúrgico, em que falhas no projeto podem acarretar no óbito do paciente. Este trabalho teve produções em paralelo em diferentes partes do projeto, tanto na construção do hardware quanto no software da aplicação de controle e da comunicação, buscando diminuir o tempo de entrega e mantendo a qualidade do projeto.

\footnotetext{
${ }^{1}$ Fundado em 2016, o Laboratório de Automação para a Vida, localizado no Instituto Federal de São Paulo, tem o intuito de prosseguir com pesquisas na área de bioengenharia ao desenvolver e aplicar soluções de automação para a melhoria e a compreensão da vida, considerando seus múltiplos aspectos. (LAV, 2016)
} 
O objetivo específico deste trabalho é desenvolver um software de visualização e controle, aplicável a um dispositivo móvel e sem fio para que centralize todas as informações dos sensores de uma máquina de circulação extracorpórea, a fim de permitir ao operador um melhor controle do fluxo de sangue e visualização em tempo real dos status do paciente, bem como propiciar mobilidade e ergonomia ao usuário.

BSC - Bombas de sangue centrífugas. O coração funciona como uma bomba muscular que promove o fluxo de sangue para todo o corpo. As doenças relacionadas com o coração são as que mais fazem vítimas no Brasil. Dados preliminares da Sociedade Brasileira de Cardiologia (SBC), mostram que no primeiro semestre de 2020, o Brasil registrou mais de 230 mil mortes, com estimativa de chegar a 400 mil no fim do ano, devidas à doenças cardiovasculares ou a causas relacionadas a elas, sendo, juntamente com as afecções do coração e da circulação, a principal causa de mortes no Brasil. Comparado ao Covid-19, uma doença infecciosa causada por um coronavírus recém-descoberto, as mortes relacionadas ao coração, apenas no primeiro semestre, foram suficientes para ultrapassar o pior cenário possível de mortes pela doença no ano de 2020.

Porém mesmo com esse número crescente de mortes, os riscos e complicações da cirurgia cardíaca ainda são mais preocupantes para os pacientes do que as próprias e evidentes doenças.

Sabendo da complexidade dessas cirurgias, os avanços clínicos ao decorrer dos anos intensificaram-se demasiadamente para facilitar esses procedimentos, desde o primeiro transplante de coração do mundo que foi realizado em 1967, passando pelo revolucionário coração artificial de Jarvik e DeVries em 1982, onde foi observada a real necessidade de um dispositivo de assistência circulatória, utilizado até os dias atuais, onde as bombas centrifugas implantáveis e as bombas de fluxo são amplamente utilizadas (Syncardia, 2020).

A bomba do presente trabalho, cedida pelo Laboratório de Automação para a Vida (LAV) é centrífuga de forma espiral, Spiral Pump®, Figura 1, que presta assistência no procedimento hospitalar, utilizada para bombear o sangue em cirurgias de tórax aberto. As bombas centrífugas surgiram como uma opção para a Circulação Extracorpórea (CEC) realizando o bombeamento de sangue em rotações mais baixas que as bombas de fluxo, obtendo menores taxas de hemólise, além de ter uma boa implantabilidade e vida estimada superior a dois anos (NOSÉ et al, 1999).

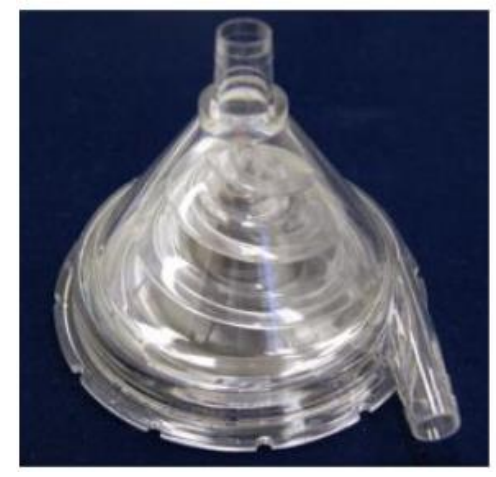

Figura 1. Spiral Pump®. 
Sistema de Controle - Blood Flow. O equipamento disponibilizado pelo LAV para o presente trabalho é um sistema de controle para dispositivos de assistência ventricular, composto por um microcontrolador responsável pela parte de envio, recebimento e manipulação de dados, por um motor que exerce o torque da bomba, recebendo sinais de controle e realizando força mecânica para gerar movimento da bomba centrífuga, que por sua vez, é responsável por converter a energia mecânica recebida do motor em vazão sanguínea (Tademos \& San Juan, 2019).

Esse sistema tem como função possibilitar a um Perfusionista ${ }^{2}$ controlar a vazão sanguínea usando a variação de rotação do motor da bomba. O microcontrolador Arduino Mega 2560 recebe os dados do driver e exibe a velocidade em RPMs e a corrente em um display para visualização do operador. O Perfusionista determinará a rotação desejada, através da manipulação de dois potenciômetros presentes no painel do aparelho, um de ajuste grosso e um para ajuste preciso. $\mathrm{O}$ sistema possui um controlador que se incumbirá de alcançar o valor de rotação mais próximo possível do selecionado de forma rápida, assim como compensar os fatores externos de forma a manter a rotação estável no valor de set-point (Tademos \& San Juan, 2019).

Estimador de pressão e vazão arterial. O uso de sensores nos Dispositivos de Assistência Ventricular (DAV) diminuem a confiabilidade do equipamento, pois podem causar hemólise ${ }^{3}$, necessitam calibração e são suscetíveis a falha, para contornar essa limitação tem se voltado ao uso de estimadores de fluxo e pressão diferencial para monitorar os parâmetros de saída das bombas de sangue. Os estimadores, frequentemente, utilizam os parâmetros de potência elétrica e rotação dos atuadores das bombas de sangue para prover essas informações de forma não invasiva (LEÃO, 2020). Mudanças de viscosidade do sangue, interação entre coração e DAV, atritos mecânicos e inércia do sistema são fatores que podem afetar a qualidade da estimação (ALOMARI et al., 2013).

Diversos estudos e pesquisas sobre estimadores tentaram se aproximar o máximo possível do estado ideal, Tanaka et al obtiveram o erro de estimação do fluxo de $0,27 \mathrm{~L} / \mathrm{min}$ e coeficiente de correlação de 0,875 , bem como o erro de estimação da pressão diferencial de 6,77 mmHg e coeficiente de correlação de 0,775 (TANAKA et al 2003).

Este projeto implementou o estimador patenteado pelo Instituto Federal de São Paulo IFSP (LEÃO, 2015), contendo um algoritmo Look-Up Table (LUT), vide Tabela 1, onde o cabeçalho de linha representa os valores de rotação e o cabeçalho de coluna representa os valores de corrente. Os valores dessa tabela foram colhidos empiricamente e equivalem aos valores das vazões correspondentes às rotações e correntes dos eixos. Utilizou-se essa tabela como exemplo

\footnotetext{
2 Perfusionista é o profissional formado e capacitado em operar os maquinários de circulação extracorpórea, seleção dos dispositivos descartáveis em cirurgias torácicas e cardíacas (SBCEC, 2020).

${ }^{3}$ Hemólise refere-se à liberação de componentes intracelulares para o meio extracelular, principalmente do conteúdo das hemácias (glóbulos vermelhos ou eritrócitos), mas também dos trombócitos (plaquetas) e leucócitos (glóbulos brancos). (PEBMED, 2019).
} 
para os cálculos feitos por interpolação bilinear dentro do código fonte do microcontrolador para estimar a pressão e vazão oriundas da bomba.

A LUT descreve, em tabelas de duas dimensões, funções que representam sistemas, podendo ser lineares ou não lineares e sua aproximação é realizada por função: bilinear, cúbica ou spline (RUGGIERO; LOPES, 1997). A interpolação bilinear (linear de dois eixos) é uma ferramenta matemática que utiliza os quatro pontos mais próximos do ponto desejado para estimar o valor buscado. Por outro lado, a interpolação spline, utilizada na referência, é matematicamente mais complexa, tem melhor precisão, principalmente para extrapolações, por considerar maior quantidade de pontos para estimar o valor buscado.

Tabela 1. Trecho exemplo de LUT utilizado para estimação de fluxo (L/min)

\begin{tabular}{|c|c|c|}
\hline \multirow{2}{*}{ Corrente (A) } & \multicolumn{2}{|c|}{ Rotação (rpm) } \\
\cline { 2 - 3 } & $\mathbf{1 7 5 0}$ & $\mathbf{2 0 0 0}$ \\
\hline $\mathbf{0 , 1 3 7}$ & 1,9 & 0,1 \\
\hline $\mathbf{0 , 1 4 5}$ & 2,3 & 0,4 \\
\hline $\mathbf{0 , 2 0 4}$ & 4,7 & 2,9 \\
\hline $\mathbf{0 , 2 0 6}$ & 4,8 & 3 \\
\hline
\end{tabular}

Microcontrolador - ESP8266 NodeMcu. O microcontrolador ESP8266 NodeMcu, fabricado pela Chinesa Espressif Systems, é um hardware muito utilizado em sistemas atuais de automação pela sua versatilidade de aplicações e pelas tecnologias embarcadas em sua placa. O baixo custo dessa tecnologia justifica a sua popularidade entre os desenvolvedores, pois se torna uma alternativa ideal para prototipações de trabalhos diversos, tanto acadêmicos quanto comerciais, além da possibilidade de a programação ser feita em linguagem $\mathrm{C}$ pelo Arduino IDE.

Segundo as especificações do fabricante Espressif (2020), dentre as principais características técnicas do ESP8266 é possível citar sua alta durabilidade, devido à sua ampla faixa de temperatura operacional, seu processador RISC tensílico de 32 bits, que consome baixa energia e velocidade máxima de clock de 160Mhz, permitindo que cerca de $80 \%$ do processamento esteja disponível para programação e desenvolvimento de aplicativos, necessário para que os cálculos de estimador fossem feitos com exatidão.

Além do processamento, a utilização do módulo ESP8266 neste trabalho fez-se necessário pela sua vantagem em possuir comunicação Wi-Fi, que consiste em um SOC com protocolo TCP/IP, capaz de hospedar uma aplicação ou descarregar todas suas funções de redes Wi-Fi a partir de outro processador, tendo um ótimo custo $\mathrm{x}$ benefício e uma comunidade de usuários enormes ao redor do mundo que continua em constante crescimento (ROBOCORE, 2020). Por intermédio desse módulo, é realizada a comunicação IoT, estabelecendo a conexão entre os dados enviados e recebidos do aplicativo para o driver do motor. 
Comunicação - Protocolo HTTP. Para ser feita a comunicação entre os dados enviados pelo ESP8266 ao Web Server, é necessário utilizar um padrão de comunicação que se adeque ao cenário do projeto. A comunicação HTTP (Hyper Text Transfer Protocol), onde são hospedados esses dados em tempo real na Web, cumpre esse requisito satisfatoriamente.

O protocolo HTTP é a linguagem mais comum da internet global moderna, transmitindo dados de forma rápida e confiável, garantindo que os dados não sejam danificados ou perdidos no caminho, mesmo vindo do outro lado do mundo. (GOURLEY et al., 2002).

Por se tratar de uma aplicação crítica conectada a um sistema de suporte a vida, utilizouse o HTTP por garantir a integridade da informação entre os dispositivos. Para garantir a cibersegurança foi idealizado que os dispositivos que compõem o sistema se comuniquem através de uma rede Wi-Fi local protegida pelo WPA2 ${ }^{4}$, sem acesso à rede externa prevenindo, assim, acessos mal-intencionados.

A plataforma utilizada para a criação do aplicativo de controle contém bibliotecas de blocos pré-definidos para a conectividade HTTP, com métodos de comunicação de dados como: GET, POST, PUT, DELETE, que facilitam no acesso e consumo dos serviços Web.

Aplicativo de Controle e Monitoração Remoto - Kodular. O aplicativo foi construído por meio do Framework Kodular que é baseada no App Inventor, criada pelo MIT (Massachusetss Institute of Technology) com o intuito de otimizar o processo de desenvolvimento de aplicações com a utilização de programação em blocos.

O Kodular ganhou notoriedade pelo fato de ter implementado funcionalidades e ferramentas do App Inventor e agregado 128 componentes diferentes a mais, além de um editor de extensões e de blocos de comunicação Web que facilitam a conexão HTTP entre aplicativos e dispositivos diversos. Segundo a Olhar Digital (2019), o Kodular é um serviço gratuito e de código fechado com mais de 90 mil usuários ativos e mais de 220 mil projetos de aplicativos que são utilizados por mais de 600 mil pessoas todos os dias.

No desenvolvimento do aplicativo foram inseridos os blocos de comunicação HTTP em conjunto com os códigos do firmware no microcontrolador, pois este fará o lado mestre da arquitetura, enquanto o aplicativo será o cliente, fazendo requisições de tipos diferentes no modelo Web. De forma simples, o aplicativo torna-se um cliente Web para efetuar as requisições ao servidor Web implementado na placa microcontrolada.

$\mathrm{O}$ aplicativo foi desenvolvido com duas telas que atendem à necessidade do projeto. A tela de abertura, que contém a caixa de inserção do IP local do microcontrolador ESP8266 para estabelecer a comunicação e a tela de monitoramento e controle, que contém as caixas de envio de rotação e Pressão Arterial Média (PAM) desejadas, bem como as caixas de leitura da rotação, vazão e pressão, vindas do microcontrolador.

\footnotetext{
${ }^{4}$ WPA2 é um padrão de segurança para redes sem fio com base na tecnologia Advanced Encryption Standard (AES). Ele é usado com os padrões IEEE 802.11a, 802.11b, 802.11g, 802.11ne 802.11ac WiFi, criptografando dados a fim de evitar acessos não autorizados.
} 
Ambiente de Simulação - Proteus. Devido às atuais condições da pandemia causada pelo Covid-19, tornou-se imprescindível a simulação de todo o equipamento disponibilizado pelo LAV, que inclui a máquina de circulação extracorpórea, a Spiral Pump® e o motor. Para tal, foi montado um ambiente dentro da plataforma Proteus, contendo os equipamentos acima citados.

De acordo com a fabricante LabCenter (2020), o Proteus combina facilidade de uso com um poderoso conjunto de recursos para permitir o design rápido, teste e layout de placas de circuito impresso profissional, com mais de 30 anos de desenvolvimento constante, o Proteus é uma das ferramentas de PCB e simulação mais produtivas e econômicas do mercado.

Modo de Operação - Blood FlowIoMT. O Laboratório de Automação para a Vida (LAV) disponibilizou a máquina de circulação extracorpórea Blood Flow com o intuito de melhoria no modo de operação do processo propondo uma modernização do conjunto por meio de dispositivos IoT. A máquina funciona com uma bomba espiral centrífuga, controlada por um driver eletrônico. Um microcontrolador Arduíno foi acoplado para controlar a rotação da bomba, bem como realizar leituras de correntes fornecidas pelo driver. Através de uma entrada no Arduíno via potenciômetro é feito o controle da rotação e a corrente é dada pelo sensor de efeito Hall do motor Brushless, ambas podem ser monitoradas por um display LCD na parte externa da máquina, como mostra a Figura 2.

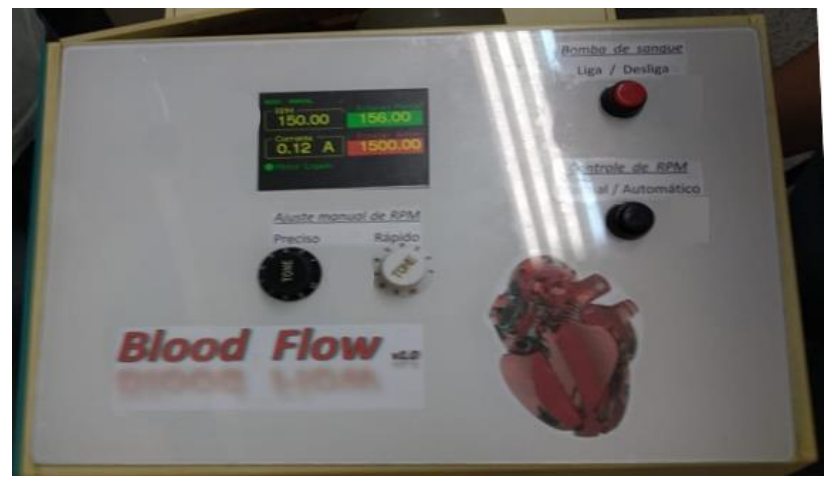

Figura 2. Tela Blood Flow.

O conjunto motor, driver, bomba e microcontrolador Arduíno foi integrado a um outro microcontrolador, o ESP8266. Esse segundo microcontrolador é utilizado para realizar os cálculos de vazão e pressão e da comunicação Wi-Fi com o aplicativo móvel. Dessa forma, o App torna-se uma interface de monitoramento e controle remoto, com campos de inserção de valores para serem enviados do aplicativo ao Arduíno, por intermédio do ESP8266, que controla o driver do motor.

Conforme Figura 3, para que se inicie o modo de operação é necessário que a conexão do microcontrolador à rede local seja pré-estabelecida e o requisitante tenha o aplicativo Blood FlowIoMT previamente instalado em seu dispositivo móvel Android. 


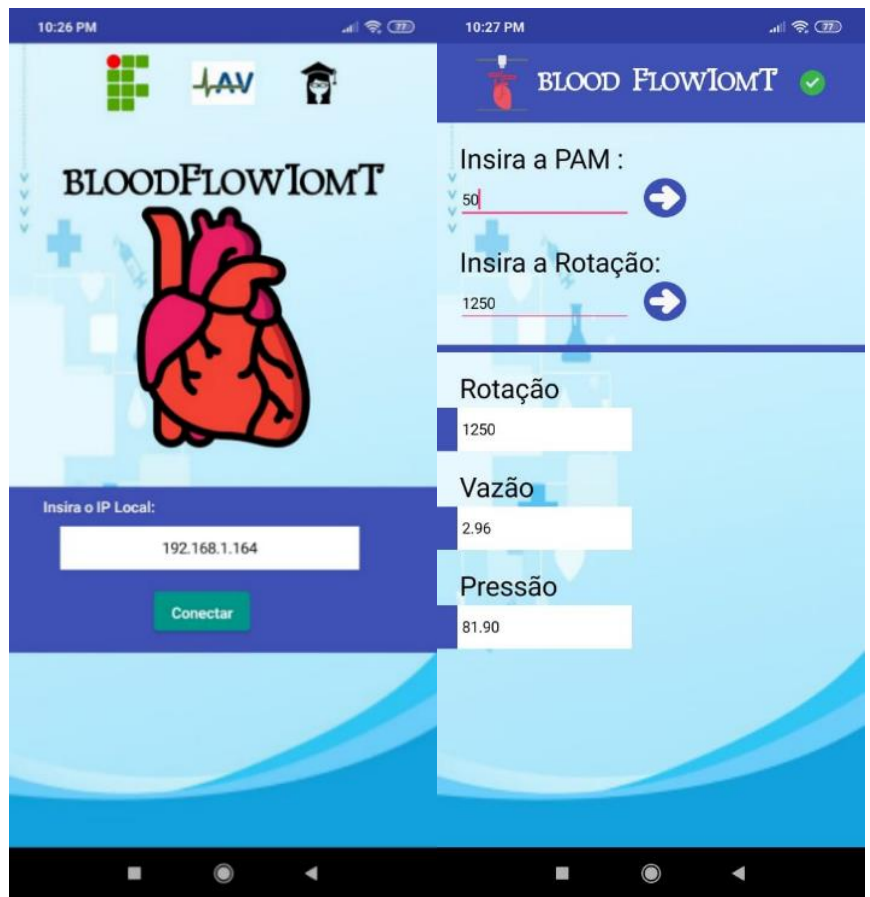

Figura 3. Telas do App Blood FlowIoMT.

Com a instalação dos requisitos exigidos, se faz obrigatória a inserção do IP local do ESP8266 na mesma faixa da rede local na tela de abertura do aplicativo que, por sua vez, fará o título do App ficar em verde e leva o usuário para a segunda tela, caso a conexão seja realizada com sucesso. Se a conexão falhar num primeiro momento, uma mensagem com o código de erro será apresentada na tela deixando o título vermelho, indicando que o IP local deve ser conferido e alterado ou a conexão Wi-Fi não esteja configurada. Ainda na tela inicial, os três ícones na parte superior direcionam o usuário respectivamente para as páginas do Instituto Federal, LAV e à documentação do presente projeto.

$\mathrm{Na}$ segunda tela, o usuário opera de fato o sistema com acesso aos valores de rotação, vazão e pressão, assim como a inserção dos valores de pressão arterial média (PAM) e a rotação desejadas. A rotação lida na segunda tela reflete diretamente o valor de saída do sensor do motor, já a vazão e a pressão são estimadas utilizando a técnica desenvolvida pelo Prof. Leão (2015). No entanto, como a LUT possui finitos pontos, utilizou-se de uma solução com interpolação bilinear para calcular os valores nos semiplanos entre pontos, relacionado aos valores da corrente lida pelo sensor Hall e da rotação. A Eq.1. expressa a interpolação bilinear da vazão e da pressão.

$$
\begin{aligned}
Q(x, y) \approx & \frac{1}{(x 2-x 1)(y 2-y 1)}\left(f\left(Q_{11}\right)\left(x_{2}-x\right)\left(y_{2}-y\right)+\left(f\left(Q_{21}\right)\left(x-x_{1}\right)\left(y_{2}-y\right)\right.\right. \\
& \left.+f\left(Q_{12}\right)\left(x_{2}-x\right)\left(y-y_{1}\right)+f\left(Q_{22}\right)\left(x-x_{1}\right)\left(y-y_{1}\right)\right) .
\end{aligned}
$$


Onde Q é o valor de pressão ou vazão correspondente à rotação e corrente reais, x2 e y2 são os valores de rotação e corrente imediatamente superiores aos valores reais na tabela, x1 e y1 são os valores imediatamente inferiores respectivamente, enquanto x e y são os valores de corrente e rotação lidas pelo sensor. $Q_{n m}$ é o valor de saída das tabelas onde n representa os índices do eixo de rotação x e m representa os índices do eixo de corrente y.

Ainda na segunda tela, na parte superior, a Rotação e a PAM digitadas pelo perfusionista são enviadas para o microcontrolador, utilizando-se de métodos de requisição do protocolo HTTP. A rotação é enviada diretamente para a entrada do driver do motor, enquanto a PAM é somada ao diferencial estimado da pressão, para que seja mostrada a pressão atual do paciente. $\mathrm{O}$ diagrama da Figura 4 resume o ciclo de operação descrito acima, assemelhando-se a uma malha fechada.

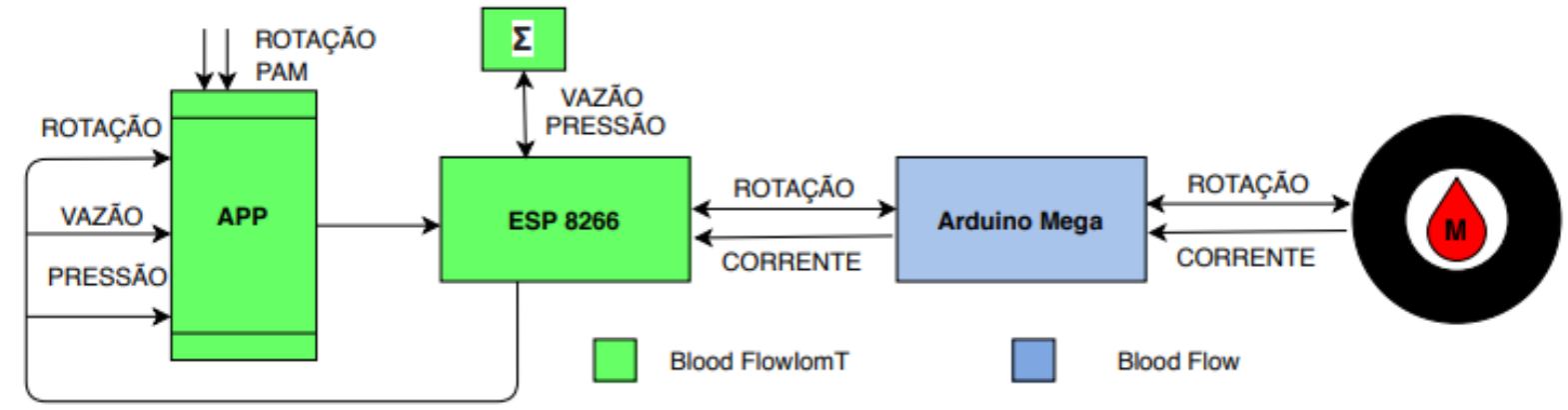

Figura 4. Diagrama de Operação.

Metodologia de desenvolvimento. O trabalho em questão é um estudo de desenvolvimento, de acordo com os objetivos propostos, de caráter construtivo e com abordagem prática. O projeto foi desenvolvido por etapas, ou seja, as partes do programa, sejam elas a programação do Arduino, do ESP8266 e do Kodular são facilmente distinguíveis, pois foram versionadas e estão divididas em arquivos separados, para que futuros grupos de pesquisa tenham a possibilidade de alterar partes isoladas do programa sem danificar as demais.

As etapas de organização e testes do projeto podem ser vistas na Figura 5, onde cada bloco de decisão representa um nível de desenvolvimento em que seu funcionamento será validado por testes antes de avançar para a próxima etapa. Caso não funcione, a etapa será revisada e testada novamente, de bloco em bloco, até o término do projeto. 


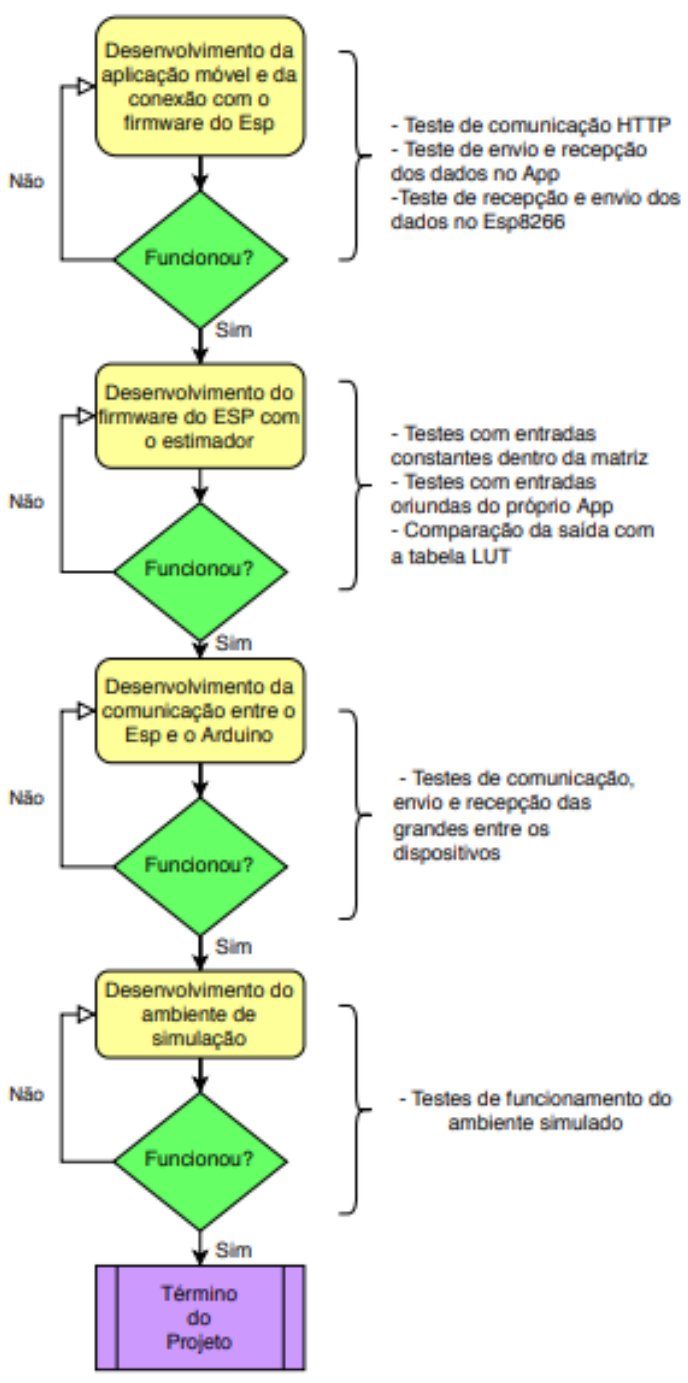

Figura 5. Fluxograma de desenvolvimento.

Cada etapa descrita no fluxograma passou por uma bateria de testes de verificação para só assim integrar ao restante do projeto. Sempre que um passo fosse concluído e verificado, iniciava-se o próximo passo, por exemplo, após a construção e os testes de funcionamento do aplicativo comunicando ao ESP8266, o foco passou a ser o desenvolvimento do estimador dentro do próprio firmware, utilizando as entradas criadas anteriormente dentro do aplicativo, caso a etapa de desenvolvimento do aplicativo não tivesse sido desenvolvida, os testes não sairiam como esperado.

Testes. Foi necessário desenvolver um ambiente que simulasse o Blood Flow físico e refletisse os valores reais de campo, bem como gerar comparações entre o presente estudo e outros já realizados com abordagem semelhante. Os testes ocorreram durante um período de 
aproximadamente um mês, dentre os quais correções na comunicação do ESP8266 externo e do Arduino emulado tiveram que ser realizados em concordância com as alterações que eram realizadas na simulação pelo software Proteus.

Em concordância com o fluxograma de desenvolvimento, os resultados foram obtidos a partir de cada etapa testada de forma modular para que o fluxo fosse criteriosamente seguido tornando-se funcional por completo. Dessa forma, os resultados foram surgindo em consonância com os testes após cada passo e concluídos quando os valores enviados e recebidos pelo App refletiam exatamente os valores demonstrados no ambiente simulado de forma integrada.

Existem diferenças específicas entre testes modulares e integrados. Nos modulares os testes são feitos de maneira isolada, por partes, buscando erros locais, enquanto os integrados são feitos com a soma dos modulares, integrando-os em um único módulo, sendo então mais complexos por abranger o sistema inteiro. Para ambos os testes é necessário entrar com informações pré-definidas e esperando saídas previstas com constantes que podem ser facilmente calculadas.

Resultados dos testes modulares. Os testes modulares acompanharam todo o desenvolvimento do projeto, toda etapa construída era seguida de um teste modular para integração ao todo. $\mathrm{O}$ App foi desenvolvido e testado inúmeras vezes, devido a facilidade da plataforma em gerar o arquivo executável (arquivo com extensão do tipo .apk).

A comunicação entre o aplicativo e o ESP8266 foi feita a partir de um código-fonte simplificado criado no IDE do microcontrolador para que ele faça o papel de servidor, enquanto o aplicativo realiza solicitações como um cliente HTTP. Primeiro o App (cliente) envia uma requisição para obter os valores de rotação, vazão e pressão vindas do ESP8266 (Server). Após esse primeiro teste, o App envia uma requisição com valores de PAM e rotação. Dessa forma, o teste foi validado com a comparação entre os valores de entrada do aplicativo sendo refletidos no Web Server aberto no browser como forma de comprovação.

Para validar os testes modulares do estimador, foram escolhidas amostras dos resultados obtidos das interpolações, conforme Tabela 2 e Tabela 3, ambas são compostas por três subtabelas: valor estimado, simulado e o comparativo percentual entre elas. Os eixos horizontal e vertical representam as rotações e correntes, respectivamente, delimitando regiões nos planos formados pelas LUTs, na de pressão a região corresponde ao centro do plano, e na de vazão corresponde a borda. O valor intermediário de $1900 \mathrm{rpm}$ foi escolhido por se encontrar entre os valores das extremidades advindos da LUT. Na tabela de valor estimado, os valores das colunas de 1759 rpm e 2006 rpm foram colhidos de forma empírica, enquanto os valores intermediários representam o cálculo com interpolação spline da tese referência.

Nas tabelas de valor simulado, temos a estimação dos valores intermediários com interpolação bilinear, possibilitando calcular a diferença percentual de menor e maior discrepância entre os resultados obtidos com interpolação bilinear e spline na terceira tabela. 
Tabela 2. Comparação entre interpolações de Vazão

\begin{tabular}{|c|ccc|ccc|ccc|}
\hline \multirow{2}{*}{$\begin{array}{c}\text { CORRENTE } \\
(\mathbf{A})\end{array}$} & \multicolumn{4}{|c|}{ Valor Estimado } & \multicolumn{6}{c|}{ Valor Simulado } & Razão Percentual de Comparação \\
\cline { 2 - 9 } & $\mathbf{1 7 5 9}$ & $\mathbf{1 9 0 0}$ & $\mathbf{2 0 0 6}$ & $\mathbf{1 7 5 9}$ & $\mathbf{1 9 0 0}$ & $\mathbf{2 0 0 6}$ & $\mathbf{1 7 5 9}$ & $\mathbf{1 9 0 0}$ & $\mathbf{2 0 0 6}$ \\
\hline $\mathbf{0 , 1 4 5}$ & 2,393 & 1,169 & 0,42 & 2,393 & 1,267 & 0,42 & 0 & $-7,76 \%$ & 0 \\
$\mathbf{0 , 2 0 4}$ & 4,783 & 3,912 & 2,901 & 4,783 & 3,709 & 2,901 & 0 & $5,48 \%$ & 0 \\
$\mathbf{0 , 2 0 6}$ & 4,864 & 3,99 & 2,959 & 4,864 & 3,777 & 2,959 & 0 & $5,64 \%$ & 0 \\
\hline
\end{tabular}

Tabela 3. Comparação entre interpolações de Pressão

\begin{tabular}{|c|c|c|c|c|c|c|c|c|c|}
\hline \multirow{3}{*}{$\begin{array}{c}\text { CORRENTE } \\
\text { (A) }\end{array}$} & \multicolumn{3}{|c|}{ Valor Estimado } & \multicolumn{3}{|c|}{ Valor Simulado } & \multicolumn{3}{|c|}{ Razão Percentual de Comparação } \\
\hline & \multicolumn{9}{|c|}{ ROTAÇÃO (rpm) } \\
\hline & 1759 & 1900 & 2006 & 1759 & 1900 & 2006 & 1759 & 1900 & 2006 \\
\hline $\mathbf{0 , 1 4 5}$ & 70,80 & 84,83 & 95,88 & 70,80 & 84,51 & 95,88 & 0 & $0,38 \%$ & 0 \\
\hline 0,204 & 70,00 & 77,64 & 95,42 & 70,00 & 78,41 & 95,42 & 0 & $-0,98 \%$ & 0 \\
\hline 0,206 & 60,33 & 77,31 & 92,00 & 60,33 & 78,11 & 92,00 & 0 & $-1,04 \%$ & 0 \\
\hline
\end{tabular}

Os valores das extremidades da tabela de razão percentual têm diferença igual a zero pois são previstos nas LUTs, sendo idênticos nas outras duas tabelas, enquanto nos intermediários pode-se notar a diferença entre os valores interpolados pelas diferentes técnicas de interpolação. $\mathrm{Na}$ tabela de vazão podemos notar uma diferença próxima a máxima pois os valores se encontram na borda e a interpolação spline utiliza mais pontos para seguir a curva, sendo mais precisa perto das bordas do plano do que a bilinear, na tabela de pressão podemos notar uma diferença próxima de zero pois ambas estão trabalhando em uma região distante das bordas.

Na última parte modular foi criado o ambiente de simulação contendo o Arduino e os outros elementos, onde foi possível validar em testes; o tratamento, a visualização e controle dessas grandezas por meio do display e dos potenciômetros, replicando, de forma simulada, a máquina referência deste projeto. A comunicação entre o ESP8266 e o Arduino se deu pela porta serial utilizando-se a arquitetura mestre-escravo e para que esse teste fosse validado, foram criadas constantes nos dois softwares, no ESP8266 físico e com o Arduino simulado no Proteus, com o intuito de verificar se os valores estavam sendo transmitidos e recebidos entre os dois dispositivos.

Resultados dos testes integrados. Após a realização dos testes modulares, o próximo passo foi integrar todo o sistema no ambiente de simulação, conforme Figura 6. Para tal, foi conectado o ESP8266 físico ao Arduino simulado dentro do Proteus com a finalidade de executar o modo de operação do conjunto completo, conforme o diagrama de operação da Figura 4.

Implementou-se uma chave para comutação dos modos de operação: local/ambiente físico e remoto/aplicativo. Para a validação dos valores de rotação mostrados no aplicativo (remoto), foram feitas comparações com os valores de rotação do ambiente referência (local) mostrados no display, constatando a proximidade entre ambos. Em seguida, realizou-se a validação dos valores estimados de vazão e pressão mostrados no aplicativo, comparando com os 
valores das LUTs disponibilizadas pela tese referência e das interpolações dos testes modulares, constatando a proximidade entre ambos.

Por conta de limitações com relação à taxa de amostragem do software Proteus foi detectada uma pequena latência, referente aos valores de rotação e corrente, interferindo, de forma insignificante, a apresentação dos cálculos das grandezas, pois se torna pouco perceptível durante as alterações de valores na simulação. No ambiente fisíco a única latência que haveria seria a de comunicação entre os microcontroladores na rede local, pois os equipamentos estariam conectados na mesma rede Wi-Fi. Portanto, a latência do sistema só pode ser medida com a aplicação em ambiente real.

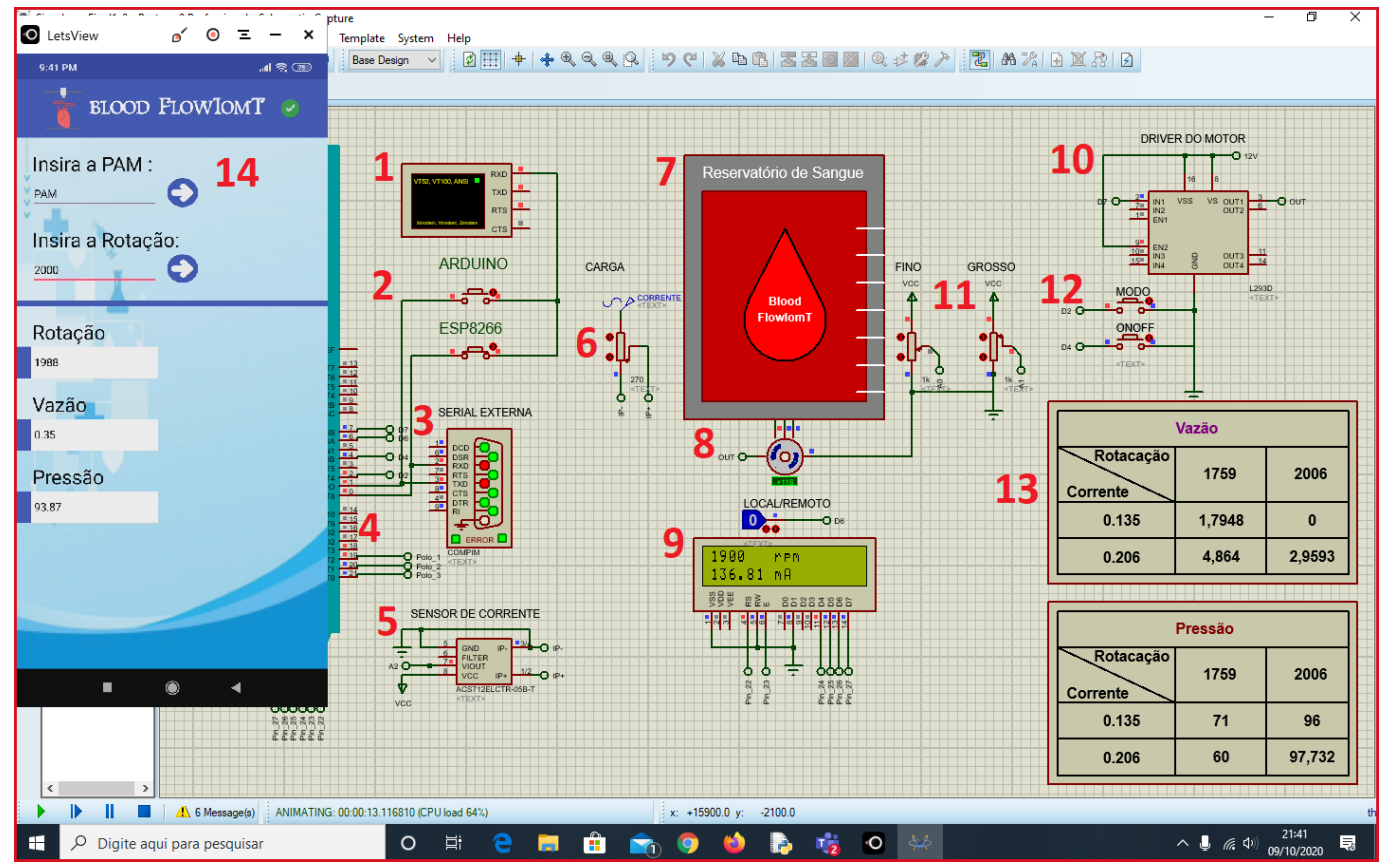

Figura 6. Ambiente de Simulação.

Legenda: 1. Monitor serial: oferece visualização das informações transmitidas pela porta serial, utilizada nos testes modulares; 2. Chaves comutadoras: selecionam a transmissão do aparelho de mesmo nome (ESP8266 ou Arduino) para ser exibida no monitor serial; 3. Porta COM: porta virtual que conecta o ambiente de simulação ao ESP8266 físico; 4. Arduino Mega 2560: microcontrolador Arduino virtualizado no ambiente; 5. Sensor de efeito Hall: tem a função de converter o sinal de corrente advindo do motor da bomba centrífuga espiral para o Arduino; 6. Simulador de carga: tem a função de ajustar a carga que é transmitida para o motor; 7. Reservatório de sangue: ilustração que representa o reservatório de sangue da máquina; 8. Motor Brushless: motor da bomba da máquina CEC; 9 . Display 2x16: exibe os valores de corrente e a rotação medidos no motor; 10. Driver do motor: controla a rotação do motor de acordo com o sinal vindo do Arduino; 11. Potenciômetros de ajustes: são dois potenciômetros para ajuste fino e ajuste grosso da rotação do motor; 12. Chaves de operação: uma das chaves tem a função de comutar o modo de operação da máquina (manual/automático) e a outra é a chave de liga/desliga; 13. Tabelas: tabelas com os cálculos do estimador; 14. Aplicativo: aplicativo móvel para monitorar e controlar o motor. 
Conclusão. A transformação digital trouxe grandes benefícios para vários campos da indústria moderna e as tecnologias baseadas em IoT foram responsáveis por muitos avanços na área médica. Logo, esses avanços impulsionam muitos desenvolvimentos e projetos importantes que mudam a forma com que interagimos com equipamentos para operações de risco ou em ambientes hospitalares, como é mostrado nesse estudo, por exemplo.

Por meio dos testes executados no ambiente simulado, comprovou-se a eficiência frente ao modelo anteriormente existente. O firmware desenvolvido é capaz de monitorar e controlar o estado do motor da máquina de forma remota, constatando um ganho no processo de operação do sistema. Além de manipular a rotação, como o projeto de antes, agora pode-se ter acesso às grandezas de pressão e vazão calculadas com o estimador implementado de forma genuína no firmware do microcontrolador utilizado neste trabalho. Constatou-se também a melhoria da máquina de circulação extracorpórea gerada pelo retrofit, justificando que este estudo deixa a possibilidade de execução de trabalhos ou testes de implementação no modelo físico.

Por mérito da aplicação dos pilares do IoMT, os objetivos foram atingidos de acordo com a proposta inicialmente estabelecida, possibilitando mudanças significativas para o profissional perfusionista exercer as suas funções com mobilidade e facilidade durante os procedimentos críticos envolvidos no ambiente cirúrgico.

Dessa forma, este trabalho colabora para que trabalhos futuros possam avançar no desenvolvimento de controles mais modernos com robustez e redundância de hardware com taxas de amostragens elevadas, agregando confiabilidade, bem como o aperfeiçoamento de segurança cibernética do sistema e até da construção de um modelo mais completo englobando todas as operações e grandezas envolvidas no procedimento cirúrgico centralizadas em uma única plataforma.

Agradecimentos. Os reconhecimentos pelo suporte são destinados ao Instituto Federal de São Paulo (IFSP) e ao Laboratório de Automação para a Vida (LAV).

Divulgação. Os autores declaram não haver conflitos de interesse neste trabalho.

Referências. (1) ALOMARI, A. et al. Developments in control systems for rotary left ventricular assist devices for heart failure patients: a review. Physiological Measurement, 1, 2013.

(2) BOCK, E. Projeto, Construção e Testes de um Dispositivo de Assistência Ventricular: Bomba de Sangue Centrífuga Implantável. Campinas: Universidade Estadual de Campinas UNICAMP [Tese de Doutorado]. 2011a.

(3) DIMITROV, D. V. (2016). Medical internet of things and big data in healthcare. Healthcare informatics research, 22(3), 156-163.

(4) ESPRESSIF. (2020). Acesso em 10 de Julho de 2020, disponível em Espressif Products: https://www.espressif.com/en/products/socs/esp8266

(5) GOURLEY, D. et al. HTTP: the definitive guide. [S.1.]: "O’Reilly Media, Inc.”, 2002. 
(6) GRAND VIEW RESEARCH. (2019). IoT in Healthcare Market Growth \& Trends. Acesso em 27 de Julho de 2020, disponível em SBC: https://www.grandviewresearch.com/pressrelease/global-iot-in-healthcare-market

(7) KODULAR. (2020). Creator. Acesso em 10 de Agosto de 2020, disponível em KODULAR: https://www.kodular.io/

(8) LAV - Laboratório de Automação para a Vida (2020). Acesso em 27 de Julho de 2020, disponível em LAV: https://lavspoifsp.wordpress.com/

(9) LEÃO, T. F. (2015). Técnica de controle automático da rotação de bombas de assistência ventricular. Tese (Doutorado em Ciências) - Universidade de São Paulo, São Paulo, 2015.

(10) LEÃO, T, Utiyama, B, Fonseca, J, Bock, E, Andrade, A. In vitro evaluation of multi-objective physiological control of the centrifugal blood pump. Artif Organs. 2020; 44: 785- 796. https://doi.org/10.1111/aor.13639

(11) NFON. (2020). WPA2. Acesso em 12 de Outubro de 2020, disponível em NFON: https://www.nfon.com/en/service/knowledge-base/knowledge-base-detail/wpa2

(12) NOSÉ, Y. et al. Development of a totally implantable biventricular bypass centrifugal blood pump system. Ann Thorac Surg, 68, n. 775-779, 1999.

(13) OLHAR DIGITAL. (2019). Kodular: crie aplicativos para Android mesmo sem saber nada de programação. Acesso em 15 de Setembro de 2020, disponível em Olhar Digital: https://olhardigital.com.br/noticia/kodular-crie-aplicativos-para-android-mesmo-sem-saber-nadade-programacao/81286

(14) PEBMED. (2019). Hemólise: quais as possíveis causas e como identificar. Acesso em 10 de Novembro de 2020, disponível em PEBMED: https://pebmed.com.br/hemolise-quais-aspossiveis-causas-e-como-identificar/

(15) ROBOCORE. Módulo Wi-Fi - ESP8266 (2020). Acesso em 15 de Setembro de 2020, disponível em Robocore: https://www.robocore.net/loja/iot/modulo-wifi-esp8266

(16) RUGGIERO, M.; LOPES, V. Cálculo Numérico - Aspectos Teóricos e Computacionais. São Paulo: Makron Books, 1997.

(17) SBC. (2020). Sociedade Brasileira de Cardiologia. Acesso em 20 de Julho de 2020, disponível em SBC: http://www.cardiometro.com.br/default.asp

(18) SOFTWARE PROTEUS. (2020). Acesso em 20 de Setembro de 2020, disponível em Labcenter Electronics: https://www.labcenter.com/

(19) SYNCARDIA. (2020). Artificial Heart Timeline. Acesso em 28 de Julho de 2020, disponível em Syncardia: https://syncardia.com/artificial-heart-timeline/

(20) TADEMOS \& SAN JUAN. (2019). Sistema de controle para dispositivos de assistência ventricular. Trabalho de Conclusão de Curso - Instituto Federal de São Paulo, São Paulo, 2019.

(21) TANAKA, A. et al. In vivo test of pressure head and flow rate estimation in a continuousflow artificial heart. Artif Org, 27, 2003. 99 - 103.

(22) UEBELHART, B. et al. Study of a Centrifugal Blood Pump in a Mock Loop System. Artificial Organs, 37, 2013. 946 - 9. 